\title{
Study of the Double Inverter Bridge Synchronous Double-frequency Induction Heating Power Supply
}

\author{
Yameng Yang ${ }^{1}$, Hui Lu ${ }^{1,2}$ and Ping Liu ${ }^{1}$ \\ ${ }^{1}$ School of Information Engineering, Zhengzhou University, Zhengzhou 450001, China; \\ ${ }^{2}$ Shangqiu Polytechnic, Shangqiu 476000, China
}

Keywords: Induction heating; Synchronous dual-frequency; Gear hardening

\begin{abstract}
For the gear with concave and convex structure such as artifacts, when using single frequency induction quenching difficulty of both convex and concave. Synchronization of double-frequency induction heating power supply overcomes the drawback of single frequency heating, can make the surface of the gear evenly heating. In this paper, the induction heating power supply and synchronization of double-frequency induction heating power supply is studied, in detail to calculate the equivalent formula of LC circuit under the condition of dual frequency, and the circuit in the simulink simulation. The simulation waveform and calculate the value of the basic agreement.
\end{abstract}

\section{Introduction}

Gear is one of the most widely used mechanical transmission parts. In order to improve the service life of gears, the need of the gear surface hardening treatment. Hardening treatment need to be in a very short time heating the surface of the workpiece, and rapid cooling, this will get over the surface of the workpiece contour of hardening layer. There are different kinds of processing way of double-frequency induction heating with heating speed is quick, convenient, accurate temperature control, the advantages of low pollution, has been widely used.

When the metal work in alternating electric current flows through the induction coil, in under the action of electromagnetic induction, metal produce eddy current, the direction of the induced eddy current and the current in the coil in the opposite direction. With the increase of coil of alternating current frequency, the skin effect will be more and more obvious, metal parts under the effect of eddy current heat surface temperature rise rapidly, thus to finish on the metal parts quenching.

\section{Role of Induction Power Supply}

Induced current in the metal pieces of the distribution is not uniform, the size of the current from inside extroversion way index decrease. According to the skin effect, the coil of alternating current frequency is higher, the current equivalent sectional area is smaller, the skin effect becomes more significant. The more complex when the shape of the metal artifacts, complete with fixed frequency surface evenly heating the less likely. For gear type of artifacts, convex and concave, it is difficult to finish on the surface of the uniform heating using a single frequency. Actual situations tend to use double frequency synchronous induction heating, the high frequency energy mainly complete heating of a crown, and the intermediate frequency heating energy is mainly used to root. So that we can make the gear surface approximate uniform heating, avoid some parts can't heating, and reach the purpose of quenching.

In order to make the gear can be heated evenly throughout the process of hardening surface, avoid convex or concave heating situation, need two frequency power energy is connected to the induction combining the way back. Main effect of intermediate frequency energy concave, high frequency energy mainly ACTS on the convex surface, two kind of frequency components of energy work together, make the heated workpieces of different position can be heated to a temperature. I

By heating the size of the workpiece and the surface on the surface of the workpiece is has the certain requirement for heating frequency. In this circuit, according to the actual situation to heating 
the workpiece, choose high frequency of $200 \mathrm{KHZ}$, intermediate frequency for $10 \mathrm{KHZ}$.

The whole circuit equivalent to two separate ac power combination to an inductive circuit, induction voltage values on the road back is the superposition of two frequency voltage value of the physical. Two power supply parts are relatively independent, can according to actual situation to adjust their frequency, power and quality factor and so on. Eventually make tooth root, crown and other parts of the gear can be evenly heating, quenching depth and effect to achieve the best state.

Intermediate frequency and high frequency energy superposition, the proportion of the two parts of the energy needed depends on the heating depth, general to heating depth is small need more high frequency energy, low frequency energy is needed for the heating depth is larger.

\section{Circuit Structure}

The whole structure of the circuit consists of four parts, rectifier, inverter, LC filter circuit and the induction coil load. Circuit structure as shown in fig. 1.

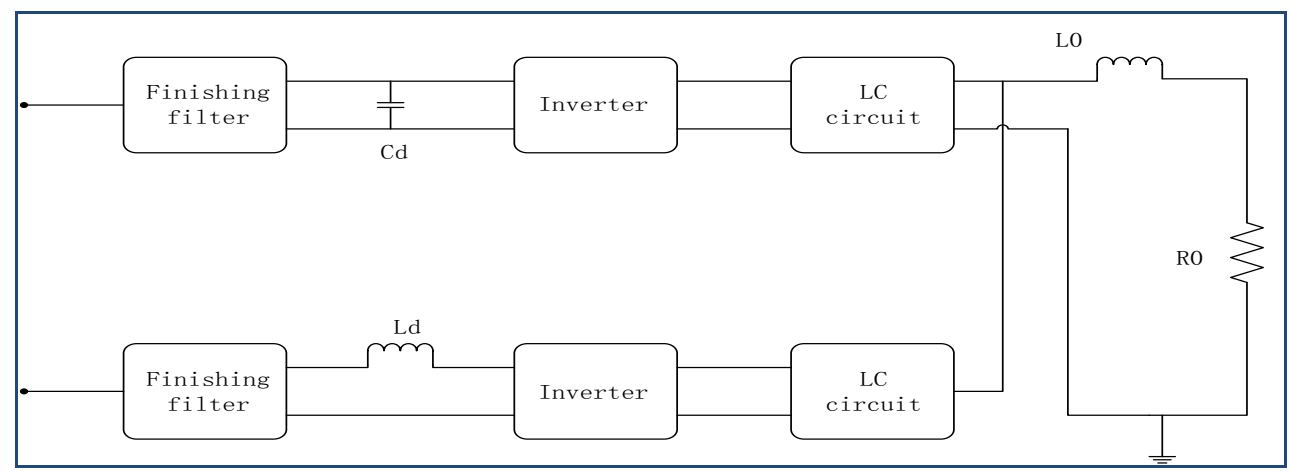

Figure 1. Circuit structure

Because of the use of double frequency power supply is not working in frequency, so the whole need to rectifier circuits, the power frequency power conversion for dc power. Based on dc power inverter, according to the requirements of the dual-band power frequency, the frequency of the inverter needed. Dual-frequency power supply circuit in the frequency selection for high frequency of $200 \mathrm{KHZ}$ and intermediate frequency $10 \mathrm{KHZ}$. The IGBT power bridge inverter circuit.. Inverter power supply with sinusoidal pulse width modulation circuit, get the approximate sinusoidal voltage source.

In order to reduce the effects of other frequency components on the circuit in the intermediate frequency and high frequency power supply output terminal access LC filter circuit, respectively. LC filter circuit diagram is shown in fig. 2. The figure will double frequency power supply with ac voltage source to replace. R0 and L0 is induction coil resistance and inductance component. In order to gain maximum energy on induction coil, need to work in every power under LC circuit in the resonance state.

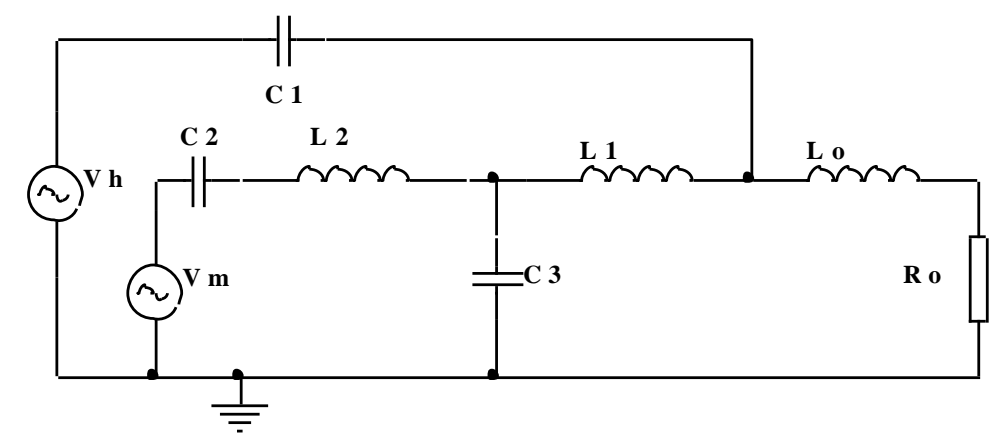

Figure 2. LC circuit

In order to simplify the circuit, the circuit of all the inductance in the selection and induction coil inductance value of the same. 


$$
L_{1}=L_{2}=L_{0}=L
$$

For the high-frequency power supply, we have

$$
\frac{1}{\omega_{h} C_{1}}=\omega_{h} L
$$

For the low-frequency power supply, we have

$$
\begin{aligned}
& \frac{1}{\omega_{m} C_{2}}=\frac{1}{2} \omega_{m} L \\
& \frac{1}{\omega_{m} C_{3}}=\frac{1}{3} \omega_{m} L
\end{aligned}
$$

According to the frequency of power supply, we have $\mathrm{C} 1=265 \mathrm{nF}, \mathrm{C} 2=212 \mathrm{Uf}, \mathrm{C} 3=318 \mathrm{uF}$.

According to figure 3 to gain the power of induction coil. Two power supply in the figure are independent of the ac power, using superposition theorem analysis alone, eventually the coil power equal to the power of a separate function of the sum of the power.

Because the circuit is complex, analyzed separately using thevenin equivalent conversion, will be equivalent to a voltage source outside of induction coil and a resistance in series form. In the process of the equivalent of two kinds of power supply, the equivalent impedance of the loop is consistent, as shown in fig. 4 , the equivalent impedance value of (use the operator to calculate):

$$
\begin{aligned}
R s=\left[\left(\mathrm{sL}_{2}+\frac{1}{s C_{2}}\right) / / \frac{1}{s C_{3}}\right. & \left.+\mathrm{L}_{1}\right] / / \frac{1}{s C_{1}} \\
& =\frac{s^{4} L_{1} L_{2} C_{2} C_{3}+s^{2}\left(\mathrm{~L}_{1} \mathrm{C}_{2}+\mathrm{L}_{1} \mathrm{C}_{3}+\mathrm{L}_{2} \mathrm{C}_{2}\right)}{s^{5} L_{1} L_{2} C_{1} C_{2} C_{3}+s^{3}\left(\mathrm{~L}_{1} \mathrm{C}_{1} \mathrm{C}_{2}+\mathrm{L}_{1} \mathrm{C}_{1} \mathrm{C}_{3}+\mathrm{L}_{2} \mathrm{C}_{1} \mathrm{C}_{2}+\mathrm{L}_{2} \mathrm{C}_{2} \mathrm{C}_{3}\right)+s\left(\mathrm{C}_{1}+\mathrm{C}_{2}+\mathrm{C}_{3}\right)}
\end{aligned}
$$

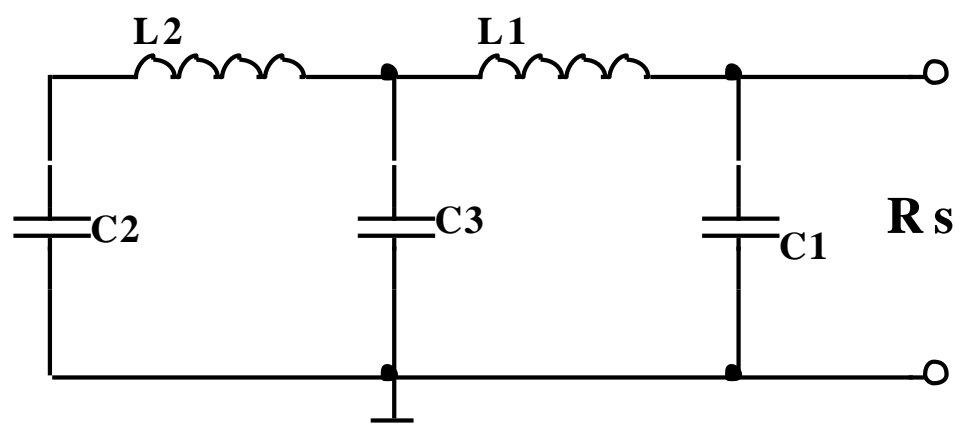

Figure 3. Equivalent impedance circuit

Because the capacitor $\mathrm{C} 1$ value is far less than the value of $\mathrm{C} 2$ and $\mathrm{C} 3$, and $\mathrm{C} 2$ and $\mathrm{C} 3$ of capacitive reactance value corresponding to the $\mathrm{C} 1$ is neglected, the impedance of the final value is approximately equal to 0 .

Under the high frequency power supply, the value of the thevenin equivalent voltage source $U_{o c h}=U_{h} \times K_{h}$, where $K_{h}=\frac{s^{2} L C}{1+s^{2} L C}$. Power of the high-frequency power supply is $P_{h}=\frac{U_{o c h}^{2}}{s L+R_{0}}$.

For the middle-frequency power supply, the value of Thevenin's equivalent voltage source is $U_{o c m}=U_{m} \times K_{m}$, where $P_{m}=\frac{U_{o c m}^{2}}{s L+R_{0}}$. For the middle-frequency power supply, the value of Thevenin's equivalent voltage source is $U_{o c m}=U_{m} \times K_{m}$, where $P_{m}=\frac{U_{o c m}{ }^{2}}{s L+R_{0}}$.

\section{Circuit simulation}

Simulink software in Matlab is used for simulation, and the sole working states of high and low-frequency voltages are simulated separately.

A high frequency power inverter input is $500 \mathrm{v}$, and the high frequency power supply working 
alone, the simulink simulation waveform diagram as shown in fig. 4. By drawing the amplitude of voltage is $1100 \mathrm{v}, 200 \mathrm{KHZ}$ frequency, current amplitude is $400 \mathrm{a}, 115 \mathrm{o}$ voltage hysteresis current, circuit work in capacitive state.

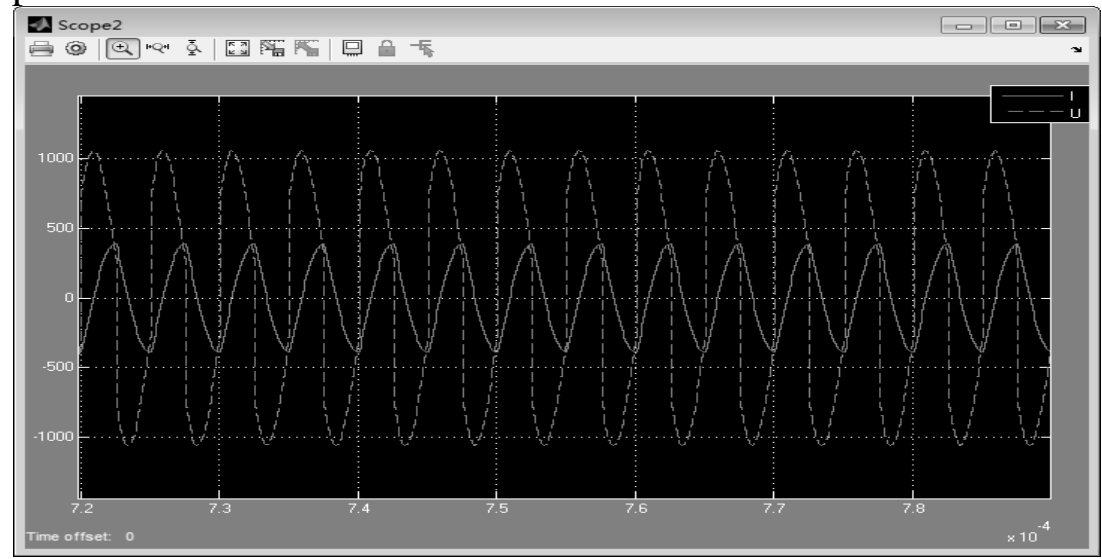

Figure 4. The sole working of high-frequency circuit

Inverter input of intermediate frequency power supply of $500 \mathrm{v}$, and the intermediate frequency power supply working alone, simulink simulation waveform diagram is shown in fig. 5. By drawing the amplitude of voltage is $2800 \mathrm{v}, 10 \mathrm{KHZ}$ frequency, current amplitude is $3600 \mathrm{~A}$, voltage hysteresis current $14.4 \mathrm{o}$, circuit work in capacitive state.

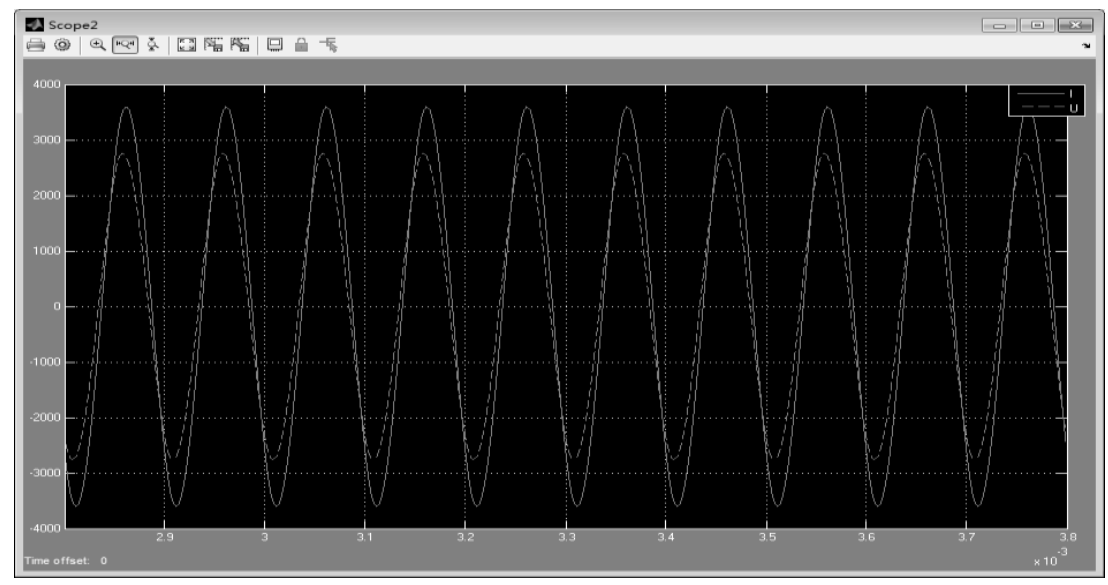

Figure 5. The sole working of middle-frequency circuit

Fig. 6 shows the output waveforms of high and low-frequency power supplies, and it can be seen from Fig. 6 that the output voltage waveforms of the two power supplies can basically meet the requirements.

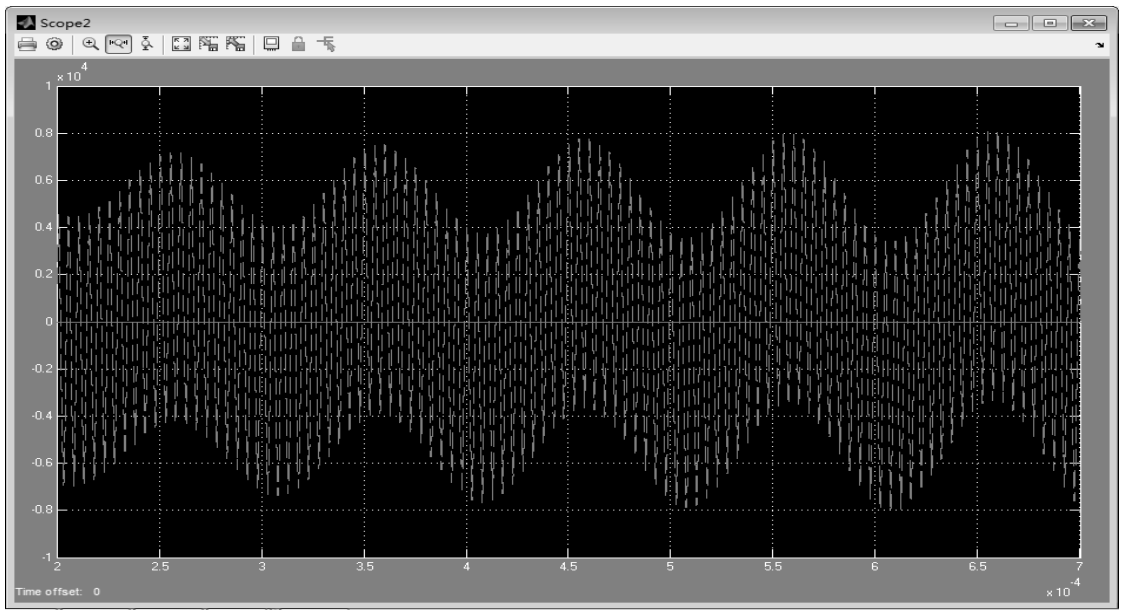

Figure 6. Simulation waveforms 
Rectified DC power supply is replaced with DC power supply for convenience.

Through the simulation waveform can know, high frequency power supply and medium frequency power supply their output waveform, amplitude, etc., each other. Because is the IGBT switching device, so there is a small amount of harmonic. I

Circuit inductance value of all values is consistent, want to change the frequency selective circuit can only change the capacitance. Through the adjustment of $\mathrm{C} 1$ and $\mathrm{C} 2$ and $\mathrm{C} 3$, found in the debugging repeatedly, parameter change affects only the power all the way. High frequency power supply and medium frequency power supply parameters are independent of each other.

\section{Conclusion}

In this paper, double-frequency inverter induction heating power supply and LC filter circuit theory analysis, simulation, and parameters of debugging. Synchronization of double-frequency induction heating compared to traditional quenching technology, small energy consumption, easy control, small footprint, large output and other advantages.

Based on simulink simulation platform, has set up a simulation for the synchronization of double-frequency induction heating circuit, by generation for different data into the simulation, the dual-band power structure and parameters of the circuit is verified the correctness of the simulation waveforms and coincided basically with the calculated value.

\section{Reference}

[1] Porpandiselvi, S, Vishwanathan, N.: Inverter configuration for simultaneous dual frequency induction hardening with independent control, Eur. Power Electron. Drives J.,2013, 23(1),PP. $12-22$

[2] $\mathrm{V}$ Esteve, $\mathbf{J}$ Jordan,E $\mathbf{J}$ Dede, et al.Induction Heating Inverter With Simultaneous Dual-frequency Ouput [A].Applied Power Electronics Conference and Exposition ,[C]2006:5.

[3] Bill Diong, Sarala Basireddy, Keith Corzine, et al. Multilevel Inverters With Equal or Unequal Sources for Dual-frequency Induction Heating [A].Applied Power Electronics Conference and Exposition ,2004[C].2004,19(2):824-830.

[4] Schwenk W.: Simultaneous Dual-frequency Induction hardening Heat treating Progress, April-May2003, pag 33-40.

[5] Dodge J.: Power MOSFET Tutorial, application note APT 0403,Advanced Power Technology, May 2004.

[6] F.J. Otto and D.H. Herring, Gear heat treatment, Heat Treat. Progr. , vol.2,pp. 30-55,jun. 2002.

[7] R. Przylucki and A. Smalcerz, Induction heating of gears-Pulsing Dual-frequency concept, Metalurgija, vol.52, no. 2,pp.190-251,April/Jun.2013.

[8] Wojda, R.P.,Kazimierczuk, M.K.: winding resistance of litz-wire and mul-strand inductors, IET Power Electron.,2012,5,(2),pp.210-255

[9] Biasutti,F.,Krause,C.,Lupi, S.: Induction hardening of complex geometry and geared parts.Heat processing,2012,vol.3,pp.26-42

[10] Hammond, M.: Simultaneous Dual-frequency gear hardening. Induction Heat Treating, June7, 2001 